\title{
Subcutaneous Solitary Fibrous Tumor: A Case Report
}

\author{
Nataliê Almeida Silva ${ }^{1}$ (D), Fausto Tambellini Oliveira ${ }^{1}$ (D), Emilia Virginia Lima Curvelo \\ Fontes $^{2}$ (D), Ulisses de Alcântara Ferreira ${ }^{3}$ (D), Lister Arruda Modesto dos Santos ${ }^{1}$ (D) and \\ Vitorino Modesto dos Santos ${ }^{*}$ (iD
}

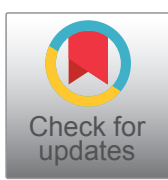

${ }^{1}$ Resident of General Surgery, Advanced Program of Oncosurgery and Videolaparoscopy, Public Workers State Hospital Francisco Morato de Oliveira, São Paulo-SP, Brazil

${ }^{2}$ Resident of Radiology, Public Workers State Hospital Francisco Morato de Oliveira, São Paulo-SP, Brazil

${ }^{3}$ Resident of Pathology, Public Workers State Hospital Francisco Morato de Oliveira, São Paulo-SP, Brazil

${ }^{4}$ Adjunct Professor of Internal Medicine, Armed Forces Hospital and Catholic University, Brasília-DF, Brazil

\begin{abstract}
We describe a 28-year-old woman with a solitary fibrous tumor at supra-pubic subcutis. The sites affected by this mesenchymal tumor include pleuropulmonary, meningeal, gastrointestinal, bones, liver, kidney, thyroid, soft tissues, and skin. More often, they occur in the head, extremities, back and shoulder. Cutaneous lesions are frequently described in the head of female individuals. Predominant histological findings include fascicles of spindle cells, staghorn-like blood vessels, and a variable amount of mitotic figures up to 10 per 10 high-power fields. Classical immunohistochemistry markers are CD34, STAT-6, CD99, and Bcl-2; while the epithelial membrane antigen and the S100-protein are usually negative. This pattern associated with the lack of adnexal entrapment constitutes the major resource in differential diagnosis with superficial tumors CD34 positive; for instance, the spindle cell lipoma, and the dermatofibrosarcoma protuberans. Follow-up care after tumor excision plays a role in the early detection of a recurrence.
\end{abstract}

Keywords

Soft tissue tumor, Solitary fibrous tumor, Subcutaneous, Superficial

\section{Introduction}

The solitary fibrous tumor (SFT) is a mesenchymal neoplasm with irregular fascicles of spindle cells, staghorn-like blood vessels, collagen, and 0 to 10/high-power fields [1-8]. This tumor more often has a low risk, even in some cases with malignant histology $[1,4]$. Reported malignancy varies up to $37 \%$ and predictive factors are high cellularity and mitotic activity, dedifferentiation, CD34 loss, and positivity for P53 and Ki67 [1-6]. Morphological variants include myxoid, fat-forming, and giant cell-rich tumors $[5,6]$. SFTs are found in people of all ages, being more often described in adult females [14]. They are more recognized in the pleuropulmonary region, and also found in meninges, gastrointestinal tract, bone, liver, kidney, thyroid, parotid, soft tissues, and skin [1-8]. Differential diagnosis with CD4-positive dermatofibrosarcoma protuberans or spindle cell lipoma may be challenging, mainly in the presence of one SFT variant $[1,2,5,7,8]$. We present the case of a young woman with unsuspected SFT located on supra-pubic subcutis, aiming to enhance the suspicion index about this very uncommon condition.

\section{Case Report}

A 28-year-old woman came to our clinic complaining of a supra-pubic nodule and increasing discomfort with local palpation, since approximately three months ago. She denied local trauma, progressive enlargement, skin changes, fever, or weight loss. There was no comorbidity, medication, previous surgery, smoking, alcoholism, or familiar antecedent of cancer. The physical examination showed a palpable nodule in the supra-pubic region of approximately $3 \mathrm{~cm}$, painful, hardened, and adhered to the deep planes. Laboratory tests were within the normal range. Chest X-ray showed absence of pulmonary calcifications or condensations, normal cardiac area, and normal pleural spaces (Figure 1). Magnetic resonance imaging (MRI) detected a subcutaneous nodular

\footnotetext{
*Corresponding author: Prof. Dr. Vitorino Modesto dos Santos, Adjunct Professor of Internal Medicine, Armed Forces Hospital, Estrada do Contorno do Bosque s/n, Cruzeiro Novo, 70658-900, Brasília-DF, Brazil, Tel:\#55-61-39662103, Fax:\#55-61-32331599 Accepted: November 24, 2020

Published online: November 26, 2020

Citation: Silva NA, Oliveira FT, Fontes EVLC, et al. (2020) Subcutaneous Solitary Fibrous Tumor: A Case Report. Dermatol Arch 4(1):95-98
} 
change measuring $2.5 \mathrm{~cm}$, with regular contours and intense contrast enhancement sited in the left para median supra-pubic area (Figure 2). Preoperative percutaneous needle biopsy

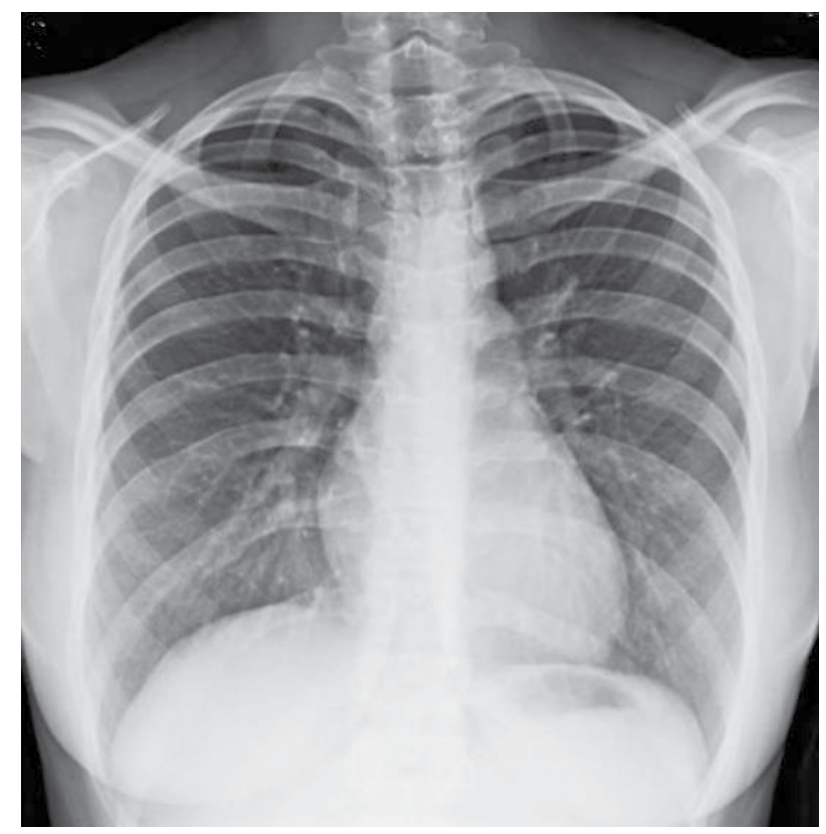

Figure 1: Chest X-ray image showing absence of abnormal pulmonary or mediastinal calcifications or condensations, normal bones, normal cardiac area, and normal pleural spaces. revealed a mesenchymal proliferation (Figure 3A). After the subcutaneous nodule excision (Figure 3B), the patient's discharge occurred on the first postoperative day. The strong nuclear STAT-6 immunoreactivity (Figure 3C) was consistent with SFT. Besides CD34 positive, immunohistochemistry data were positivity for $C D 31$, vimentin, b-catenin, CD99, ki-67 and $\mathrm{Bcl}-2$ (Figures 3D and Figure 3E); while desmin, S-100 protein, CD117 c-kit, anti-smooth muscle, and p53 were negative. The most useful immunohistochemical SFT markers were CD34, $\mathrm{Bcl}-2$ and STAT-6. The anatomopathological evaluation established the diagnosis of a subcutaneous SFT with a cell proliferation index of up to $10 \%$. The case was categorized in low risk score 2 of the malignancy risk stratification. Currently she underwent careful follow-up on an outpatient basis in the Oncology Service of the hospital, and there is no evidence either of local recurrence or metastasis after a period of approximately one year.

\section{Discussion}

SFT was reported as a pleural tumor in 1870, and later called fibrous mesothelioma [2]. Further studies confirmed the pleural prevalence and other locations of primary origin. The patient herein described had an unsuspected subcutaneous SFT affecting the supra-pubic region, which was easily excised and the postoperative course was uneventful. The MRI study showed a subcutaneous lesion with intense contrast enhancement, indicative of a quite vascularized tumor, but

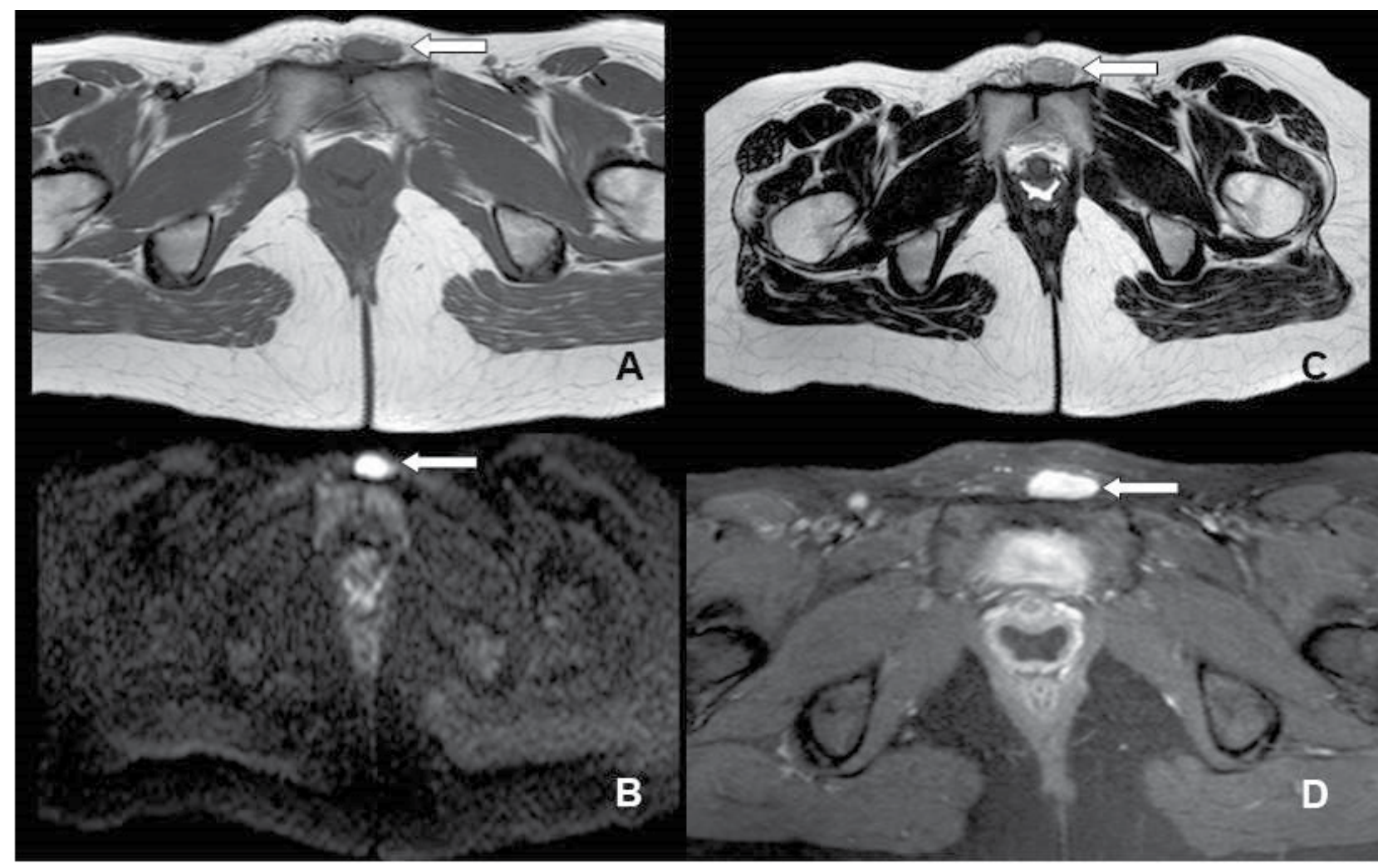

Figure 2: Magnetic resonance imaging of the pelvis in axial sections. A) T1-weighted image shows the suprapubic nodular lesion with isosignal; B) b1000 sequence of diffusion shows marked restriction to the diffusion of water molecules; C) T2-weighted image shows slight hypersignal of the lesion; and D) T1-weighted image shows intense enhancement after administration of paramagnetic contrast. 


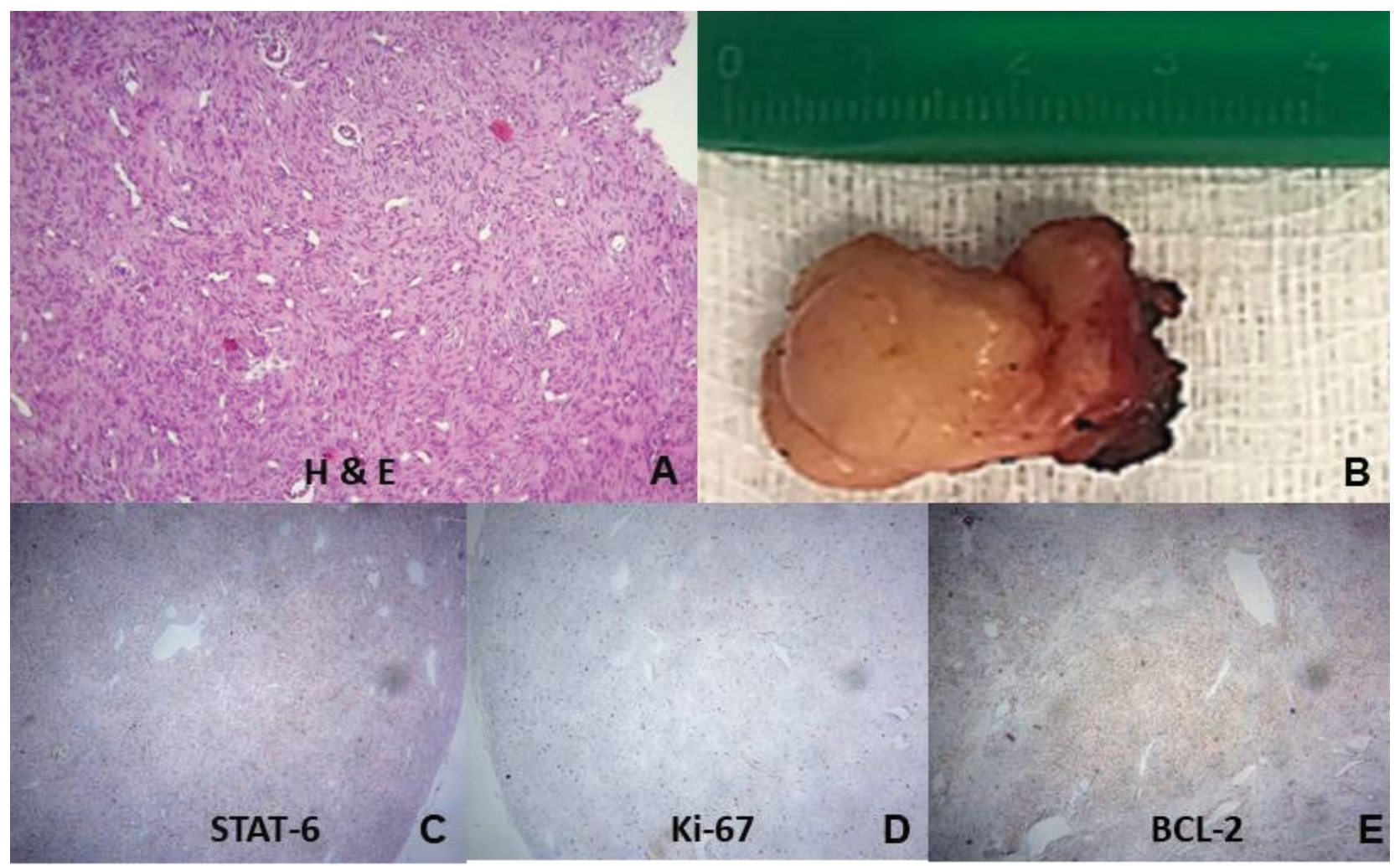

Figure 3: Photomicrography of the histopathology pattern of percutaneous aspiration biopsy sample (Hematoxylin \& Eosin, 10x magnification) compatible with a subcutaneous fibrous tumor (A); a gross aspect of the surgical specimen of the solitary fibrous tumor excised from the subcutaneous supra-pubic region (B); and immunohistochemistry images (4x magnification) showing positivity for STAT-6 (C), Ki-67 (D), and BCL-2 (E).

this finding is not specific of STFs [4]. The final diagnosis was based by classical histopathology and immunohistochemistry data. NAB2-STAT6 fusion genes are specific for SFT, but this cumbersome resource is often unavailable and the immunohistochemistry for STAT6 is utilized in daily practice [2]. The strong nuclear STAT6 immunoreactivity, highly sensitive and specific for SFT, allowed to rule out the differential diagnosis with other CD4-positive tumor [1,2,4-7]. Besides STAT6, the main tumor markers were CD34, CD99, and Bcl-2 expressions $[2,4]$. Immunohistochemistry markers for soft-tissue benign SFT include STAT6 (99-100\%), CD99 (89-100\%), CD34 (85$100 \%), \mathrm{Bcl}-2$ (85-100\%), and beta-catenin (22-67\%) [2]. Worthy of note were the rare site and tumor size in a patient with 28 years at diagnosis; and due to the indolent growing of SFT $[1,4-6]$, its origin seems to occur many years before. The total scores of malignancy risk stratification in individuals with SFT are: 0-2 (low), 3-4 (moderate), and 5-6 (high) risks [4]. The young patient herein reported had a total score 2 corresponding to the age of 28 years $=0$ point; tumor size $2.5 \mathrm{~cm}$ $=0$ point; and mitotic figures up to 10/10 high-power fields $=$ 2 points. Different from the present case, the majority of superficial STFs are on extremities [1,4]. Feasel, et al. reviewed 26 cases of superficial STFs, the mean age of patients was 47 $(16-80)$ years, $73 \%$ were women, the sites were head, thigh, back, shoulder, arm, ankle, and toe, $7.7 \%$ were malignant; and had a low-risk with a mean follow-up of 100 months [1]. Kiyohara, et al. reviewed data of six patients with malignant SFT in the head, trunk, and thigh; four were males, one tu- mor-related death occurred after excision and radiotherapy, but there was no recurrence or metastases in 16 months of follow-up [3]. Öztürk, et al. reviewed 7 cases of STFs in extremities, $57.1 \%$ in females, age ranging 22 to 79 years, tumor size ranging 8 to $15 \mathrm{~cm}$, and with the main site $(42.8 \%)$ in the thigh [4]. All patients had primary tumors evolving as localized painless masses, two of low risk group underwent marginal resection and adjuvant radiotherapy; those at moderate and high risk groups had wide resections. Although metastasis or recurrence may occur in up to $15 \%$ of STFs, they were not observed in the follow-up of these patients from 13 to 121 months [4]. Excision is the first treatment option for STFs, while radiotherapy or chemotherapy has poor results; and the course of tumors with malignant features is similar to high-grade sarcomas $[4,6]$. Future researches are needed to better understand the molecular mechanisms of STFs, allowing effective targeted treatments and good outcomes [6]. Single case reports may increase the awareness about very scarcely reported conditions.

\section{Conclusion}

This case study raises the concern about the longstanding evolution of superficial SFTs in uncommon sites and affecting people younger than the current estimated mean age. Besides, the diagnosis of an unsuspected STF depends on anatomopathological routine, including complete immunohistochemistry panel based on the nature of affected tissues. 
Incidental diagnosis of rare entities also occur and should enhance the suspicion index of SFT presenting as superficial lesion and mimicking other more common neoplasms.

\section{References}

1. Feasel P, Al-Ibraheemi A, Fritchie K, et al. (2018) Superficial solitary fibrous tumor: A series of 26 cases. Am J Surg Pathol 42: 778-785.

2. Geramizadeh B, Marzban M, Churg A (2016) Role of immunohistochemistry in the diagnosis of solitary fibrous tumor, a review. Iran J Pathol 11: 195-203.

3. Kiyohara T, Tanimura H, Takewaki H, et al. (2019) Malignant solitary fibrous tumor in the subcutis: Report of a rare superficial malignant type and review of published work. J Dermatol 46 : 267-270.
4. Öztürk R, Arıkan ŞM, Şimşek MA, et al. (2017) Management of solitary fibrous tumors localized in extremity: Case series and a review of the literature. Eklem Hastalik Cerrahisi 28: 121-127.

5. Ronchi A, Cozzolino I, Zito Marino F, et al. (2018) Extrapleural solitary fibrous tumor: A distinct entity from pleural solitary fibrous tumor. An update on clinical, molecular and diagnostic features. Ann Diagn Pathol 34: 142-150.

6. Thway K, Ng W, Noujaim J, et al. (2016) The current status of solitary fibrous tumor: Diagnostic features, variants, and genetics. Int J Surg Pathol 24: 281-292.

7. Tran TAN (2020) Primary cutaneous solitary fibrous tumor with entrapped eccrine components. J Cutan Pathol 47: 845-849.

8. Wood L, Fountaine TJ, Rosamilia L, et al. (2010) Cutaneous CD34+ spindle cell neoplasms: Histopathologic features distinguish spindle cell lipoma, solitary fibrous tumor, and dermatofibrosarcoma protuberans. Am J Dermatopathol 32: 764-768.

DOI: $10.36959 / 661 / 311$

Copyright: (c) 2020 Silva Na, et al. This is an open-access article distributed under the terms of the Creative Commons Attribution License, which permits unrestricted use, distribution, and reproduction in any medium, provided the original author and source are credited. 